\title{
HIGH REDSHIFT RADIO GALAXIES
}

\author{
K. MEISENHEIMER, H. HIPPELEIN AND M. NEESER \\ Max-Planck-Institut für Astronomie \\ Königstuhl 17, D-69117 Heidelberg
}

\section{Glancing back into the young universe}

One hundred years after G. Marconi recorded radio waves over a distance of more than $1000 \mathrm{~m}$, the most sensitive radio telescopes are able to detect the radio emission from light travel distances at least $1.4 \times 10^{23}$ times greater. The electromagnetic waves from these distant objects are red shifted by $\Delta \lambda / \lambda \equiv z>4$. It is not the mere distance of high redshift objects which is fascinating, but rather the fact that one looks back into the early history of the universe by observing them: Objects at a redshift of 4 shined at a time when the universe had reached only about $1 / 5$ of its present age.

Celebrating a century of radio transmission it is appropriate to recall briefly the history of how radio sources were identified with more and more distant objects. High redshift astronomy started back in 1960 when Minkowski identified 3C 295 with a galaxy at $z=0.46$ (at that redshift the age of the universe relative to the present age was $t / t_{0}=0.69$ ). After the detection of quasars (by M. Schmidt, 1963) and the subsequent discovery that quasars can be found out to a very substantial redshift (3C 9 at $z=2.01$, i.e. $t / t_{0}=0.32$, Schmidt 1965), the focus of interest shifted to these extremely luminous "beasts". With the development of optical CCD detectors, it also became feasible to identify the less conspicuous radio galaxies at high redshift: 3C 13 was identified with a galaxy at $z>1$ (Spinrad et al. 1981) and in $19843 \mathrm{C} 256$ was found to be a galaxy at $z=1.81$ $\left(t / t_{0}=0.36\right.$, Spinrad \& Djorgovski), revealing for the first time the strong Ly- $\alpha$ emission of these galaxies. 1988 brought a break-through in the study of high redshift radio galaxies. Within a few months of Spinrad et al. detecting the most distant object in the $3 \mathrm{C}$ catalogue (3C 257 at $z=2.48$ ), Lilly identified the source $0902+34$ from the $2^{\text {nd }}$ Bologna catalogue with a galaxy at $z=3.39$ and Chambers et al. found that $4 \mathrm{C} 41.17$ is located 
even at $z=3.80\left(t / t_{0}=0.21\right)$. The very extended Ly- $\alpha$ cloud surrounding the latter galaxy emits $10^{38} \mathrm{~W}$, comparable with a moderate quasar. With 4C 41.17 radio galaxies overtook the radio loud quasars in redshift for the first time since 1965 ! During this conference we heard that both the most distant radio galaxy $(6 \mathrm{C} 0140+32$ at $z=4.41$, Lacy et al., these proc.) and the radio loud quasar ( $\mathrm{P} 1251-407$ at $z=4.46$, Shaver, these proc.) allow us to look back into the very distant history of the universe $\left(t / t_{0}<0.2\right)$. The highest redshift found so far $(z=4.89$ for the radio quiet QSO PC 1247+3406, Schneider et al. 1991) is hardly higher than this.

But why expend so much effort in finding these distant radio galaxies when quasars are much easier to detect? Because radio galaxies appear extended in the optical/near-infrared band ! This provides (i) the opportunity to separate the stellar component from the AGN (out-shining everything in QSOs) and (ii) allows one to study the kinematics and ionization structure of the - often very extended - emission line gas. Thus, radio galaxies are our crown witnesses to early galaxy formation and evolution.

\section{Radio galaxies as a probe of galaxy evolution}

At redshifts $z \gtrsim 1$, any investigation of the stellar spectral energy distribution (SED) has to include observations in the near-infrared $(1<\lambda<$ $2.3 \mu \mathrm{m})$. In a seminal piece of work Lilly \& Longair (1984) employed $J H K$ photometry of more than a dozen 3C-galaxies at $z \simeq 1$. They found that none of the galaxies at $z \gtrsim 1$ showed the very red $R-K$ colors expected for a red shifted present-day giant elliptical. In fact, about one half of the galaxies at $z>1$ were even bluer than passive evolution (i.e. star formation at $z \gg 1$ ) would predict. The authors concluded that these galaxies contain a substantial fraction of young stars. Many show distorted optical images.

Subsequently it became even more evident that there exists a problem in using radio galaxies as a tracer for galaxy evolution in general. Two groups (Chambers et al. and McCarthy et al., 1987) reported a strong correlation between the radio axis and the orientation of the extended emission line regions (EELR) around many of the radio galaxies at $z \gtrsim 1$. In some cases this "alignment effect" even seems to be present in the orientation of the elongated blue continuum, pointing strongly to the existence of a very special radio-related component (RRC) in the SED of these powerful radio sources. As a result the overall SED of powerful radio galaxies cannot be regarded as representative for the stellar SED of galaxies in general.

On the other hand, radio galaxies show a very small scatter in the $K-z$ (Hubble-)diagram (Lilly 1989) and the aligned component becomes less conspicuous with decreasing radio power (Dunlop \& Peacock 1993). Thus, we can hope that RRC and stellar SED might eventually be separable. 


\section{High redshift radio galaxies as a laboratory to study the physics of powerful radio sources}

The radio-related component in galaxies at $z \gtrsim 1 \mathrm{might}$ provide valuable information about the interaction of the radio source with its environment. The kinematics of the EELR may show direct evidence for the protrusion of the bow-shock into the surrounding medium or for sideways and backward flow of the radio plasma. The excitation of the EELR could indicate whether the kinetic power of the jet or radiation from a (hidden) AGN dominates the energetics of the RRC. The morphology of both the EELR and the optical continuum should trace the density distribution around the galaxy and thus reveal whether the most powerful radio galaxies are caused by an atypical environment. In addition, the role of galaxy-galaxy interactions for triggering and shaping the radio source can be investigated.

\section{The radio-related component at $z \simeq 1$.}

We will not attempt to give a general discussion of the alignment effect - an excellent review of this topic has been presented by Clive Tadhunter at this conference. Rather, we will highlight some results of our ongoing project to study a dozen radio galaxies in great detail employing $2 \mathrm{D}$ spectroscopy with a Fabry-Pérot-Interferometer (FPI), supplemented by continuum imaging in line-free bands. We have studied ten $3 \mathrm{C}$ galaxies in the range $0.47<z<1.13$, selected by their very extended emission line regions: 3C 34, 44, 169.1, 265, 337, 352, 356, 368, 435 A, 441.

By discussing three examples we will demonstrate the wide variety encountered in this sample (details are given in the Thesis of Mark Neeser).

$3 C 368(z=1.13) \quad$ shows a double-lobe structure with a blue-shifted southern and red-shifted northern lobe. There is a perfect correspondence between the extent of the emission line lobes (EELs) and the radio lobes, although the latter protrude about $1^{\prime \prime}(10 \mathrm{kpc})$ further from the source (Meisenheimer \& Hippelein 1992). The southern EEL shows a conical structure which, together with the kinematical evidence for an expanding shell, can naturally be explained as external gas which has passed the bow-shocks in front of the radio lobes. Our interpretation of the ground-based FPI-data ( $~ 11^{\prime \prime} 3$ resolution) is confirmed by recent HST results (Longair et al. 1995) which - when properly aligned to the radio coordinates ${ }^{1}$ show both the 1:1 correspondence of the radio and emission line lobes and the bow-shock morphology of the southern lobe.

\footnotetext{
${ }^{1}$ Based on our astrometry, the brightest peak in the HST image (an M-star) has to be shifted to $18^{h} 05^{m} 06^{8} .429,11^{\circ} 01^{\prime} 31^{\prime \prime} 45$ (J2000), i.e. $\sim 1^{\prime \prime}$ to $\mathrm{NW}$ w.r.t. the radio map.
} 
The ELLs of 3C $368 \mathrm{lag}$ by about $10 \mathrm{kpc}$ behind the present location of the bow-shocks. This behaviour is expected since the shocks should heat the external material to $\gtrsim 10^{7} \mathrm{~K}$ and it takes even over-dense parts some $10^{7}$ years to cool down to $20000 \mathrm{~K}$ - the temperature at which $\mathrm{O}^{+}$is the most abundant ion. From this observed cooling length and the assumption that ram pressure balances the (minimum) pressure in the radio lobes, we derived a self-consistent model of $3 \mathrm{C} 368$ which is characterized by a rather high external density $n_{e x}=10^{5} \mathrm{~m}^{-3}$ and a moderate expansion velocity $v_{\text {bow }}=1200 \mathrm{kms}^{-1}$. Thus we conclude that the perfect radiooptical alignment and the high radio power of $3 \mathrm{C} 368$ are caused by a high external density with an atypically smooth distribution. Note, that this model leaves the excitation of the EELR undetermined since the bow-shocks alone cannot provide the necessary energy. Based on the exceptionally low $[\mathrm{SIII}] \lambda 953.2 /[\mathrm{OII}] \lambda 372.8$ ratio and the absence of anything resembling an radiation cone, we favour the explanation that the excitation is dominated by the interaction between the post-bow-shock gas and the cocoon of the radio source. This interpretation has gained substantial support by the fact that the high extended polarization reported for this source could not be verified with Keck or HST observations (van Breugel, this conf.).

$3 C 435 A(z=0.47): \quad$ Although this source, with its narrow northern spur of emission line gas extending $>10 \mathrm{kpc}$ beyond the radio lobes, has been called a prime witness for beamed ionizing radiation from a hidden quasar we think that the correct interpretation of the EELR of $3 \mathrm{C} 435 \mathrm{~A}$ is rather different: Most likely the morphology and kinematics of this asymmetric EELR is dominated by a violent interaction between the radio galaxy and a companion galaxy about $5^{\prime \prime}(30 \mathrm{kpc})$ towards the north (see Neeser et al. 1996). The northern spur of emission line gas much more resembles a tail of tidal debris than a randomly distributed gas ionized by a AGN beam.

3C $265(z=0.81)$ : This is the most extended and most luminous EELR around any radio galaxy with $z<1$. In spite of its large linear extent the diameter of the EELR is still less than half of the radio source. Both the complicated morphological and kinematical structure of the EELR indicate that it is a superposition of several different processes: Polarimetric evidence (Cohen, this conf.) indicates that a hidden luminous AGN is partly responsible for the ionization of the inner EELR. We derived a similar conclusion for the outermost parts. However, the peculiar velocities observed in the inner region, as well the fact that there is plenty of emission line gas under $90^{\circ}$ w.r.t. the radio axis, make it very likely that the presence of several faint gas-rich companions dominates the EELR in the innermost $50 \mathrm{kpc}$. We regard 3C 265 as an example of powerful radio sources in which galaxy interactions play a major role in supplying the material for the EELR while 
an AGN (fed by the same process) provides most of the ionizing radiation.

In summary, we conclude from our sample of $3 \mathrm{C}$ galaxies: The perfect radio-optical alignment found in the prototype $3 \mathrm{C} 368$ is not typical and is caused by a very fortunate density distribution. Galaxy-galaxy interactions are common in powerful radio galaxies at $z \simeq 1$. Since they should lead to an isotropic distribution of position angles, additional processes (anisotropic ionization from an AGN, brightening of the radio source due to interaction with a companion, etc.) are required to explain the alignment effect. We found several examples in which ionization from a hidden quasar is a likely origin of the alignment. In view of the complicated superposition of the different processes which we found in several sources, we recommend a detailed study of each individual case in order to disentangle the RRC from the typical SED of high redshift galaxies.

\section{An example at very high redshift: $4 \mathrm{C} 41.17$.}

4C 41.17 ( $z=3.80$, see Chambers et al. 1990) is the best studied radio galaxy at very high redshift. Its optical continuum is well aligned with the radio axis (Miley et al. 1992). Absolute astrometry indicates that the radio core (Carilli et al. 1993) sits exactly in the dark "gap" which is conspicuous on both Ly- $\alpha$ and continuum images taken at $0.6<\lambda<2.2 \mu \mathrm{m}$, and is most likely caused by dust absorption. Millimetre and sub-mm observations (Dunlop et al., Chini \& Krügel 1994) found a large amount of warm dust, most of which is presumably concentrated near the "gap". We have carried out FPI and long-slit spectroscopy of the Ly- $\alpha$ cloud and narrow band imaging in the [OIII] $\lambda 5007$ line which is red-shifted to $2.40 \mu \mathrm{m}$.

Our main results can be summarized as follows: Although the [OIII] image (low $\mathrm{S} / \mathrm{N}$ ratio) is consistent with the brightest parts of the Ly- $\alpha$ emission, the "gap" seems deeper in Ly- $\alpha$ than in [OIII] (Hippelein et al. 1995). This supports dust absorption as an explanation for the gap. The high-brightness Ly- $\alpha /[\mathrm{OIII}]$ region is perfectly aligned with the radio axis and might either be ionized by an UV radiation cone or young stars.

The outer isophotes of the extended Ly- $\alpha$ halo are much rounder than that of the inner high brightness region. At radii $>2^{\prime \prime}$ from the core we find substantial Ly- $\alpha$ brightness even at $90^{\circ}$ w.r.t. the radio axis, which can hardly be explained by UV radiation from an obscured core. Although resonant scattering could broaden the Ly- $\alpha$ emission region, we think that the lack of a sharp boundary between "cone" and halo argues against this interpretation. The red/blue-shift asymmetry in the eastern/western part of the EELR seems to argue for a radio-related kinematics, but the velocity field of the more extended eastern lobe indicates contraction rather than expansion. In addition, a Ly- $\alpha$ absorption at $z=3.793$, with an equivalent 
width of $0.3 \mathrm{~nm}$ covers the entire cloud (Hippelein \& Meisenheimer 1993, Hippelein et al. 1995).

Associating this absorption with 4C 41.17 (in analogy to the absorption found in other radio galaxies, see Röttgering this conf.) we propose the following interpretation: The systemic redshift of $4 \mathrm{C} 41.17$ is traced by the absorption. The blue-shifted western component (and perhaps the brightest parts in the east) are caused by the interaction of the radio source with the surrounding material, most probably ionised by a hidden quasar. The extended Ly- $\alpha$ halo, however, which is red-shifted and contracting (see Fig. 3 in Meisenheimer et al. 1994), is due to ongoing accretion of surrounding material (optically thick in Ly- $\alpha$ ) onto the density peak at which the source is located. In this scenario, violent accretion shocks would be responsible for the extreme Ly- $\alpha$ luminosity and the large velocity width. $4 \mathrm{C} 41.17$ would therefore be an extremely massive galaxy or cluster core in the process of formation. The alignment of the radio axis along the elongation of the extended halo is caused by a selection bias; only those radio sources which happen to be aligned with the density distribution will be sufficiently luminous to exceed the limit of the $4 \mathrm{C}$ catalogue. Consequently, there should be at least $10 \times$ more "misaligned $4 \mathrm{C} 41.17 \mathrm{~s}$ " with equally bright Ly- $\alpha$ haloes, but much weaker radio sources.

\section{References}

Carilli, C., Owen, F.N., Harris, D.E. 1994: Astron.J. 107, 480

Chambers, K., Miley, G., van Breugel, W. 1987: Nature 329, 604

Chambers, K., Miley, G., van Breugel, W. 1990: Astrophys.J. 363, 21

Chini, R. \& Krügel, E. 1994: Astron. Astrophys. 288, L33

Dunlop, J. \& Peacock, J. 1993: Mon. Not. R. astr.Soc. 263, 936

Dunlop, J., et al. 1994: Nature 370, 347

Hippelein, H. \& Meisenheimer, K. 1993: Nature 362, 224

Hippelein, H., Meisenheimer, K. \& Röser, H.-J. 1995: in Galaxies in the Young Universe (eds. H. Hippelein et al.), Springer Lecture Notes, p.93

Lilly, S.J. 1988: Astrophys.J. 333, 161

Lilly, S.J. 1989: Astrophys. J. 340, 77

Lilly, S.J. \& Longair, M.S. 1984: Mon. Not.R.astr.Soc. 211,833

Longair, M., Best, P., Röttgering, H. 1995: Mon. Not. R. astr. Soc. 275, L47

McCarthy, P. et. al. 1987: Astrophys. J. (Lett.) 321, LL29

Meisenheimer, K. \& Hippelein, H. 1992: Astron. Astrophys. 264, 455

Meisenheimer, K., Hippelein, H., Neeser, M. 1994: in The Physics of Active Galaxies (eds. G. Bicknell et al.), ASP conf. series Vol. 54, p.397

Miley, G., Chambers, K. van Breugel, W., Macchetto, F. 1992: Astrophys.J. 401, L69

Minkowski, R. 1960: Astrophys. J. 132,, 908

Neeser, M., Hippelein, H. \& Meisenheimer K. 1996: in preparation

Schmidt, M. 1963: Nature 197, 1040

Schmidt, M. 1965: Astrophys.J. 141, 1295

Schneider, D.P., Schmidt, M., Gunn, J.E. 1991: Astron. J. 102, 837

Spinrad, H., Staufer, J., Butcher, H. 1981: Astrophys. J. 244, 382

Spinrad, H. \& Djorgovski, S. 1984: Astrophys.J.(Lett.) 285, LL49 